\title{
Oral health and oral health-related quality of life in patients with oral dystonia indicates their need for dental special care
}

\author{
Gerhard Schmalz ${ }^{1}$, Holger Ziebolz ${ }^{2}$, Tanja Kottmann ${ }^{3}$, Dirk Ziebolz ${ }^{1}$, Rainer Laskawi ${ }^{4}$ \\ ${ }^{1}$ Department of Cariology, Endodontology and Periodontology, University Leipzig \\ ${ }^{2}$ Private dental practice, Hanau, Germany \\ ${ }^{3}$ Clinical Research Organisation, Hamm, Germany \\ ${ }^{4}$ Department of Otorhinolaryngology, Head and Neck Surgery, University of Göttingen, Göttingen, Germany
}

Correspondence:

University Leipzig, Dept. of Cariology

Endodontology and Periodontology

Liebigstr. 12, D 04103 Leipzig, Germany

dirk.ziebolz@medizin.uni-leipzig.de

Received: 23/11/2020

Accepted: 29/03/202

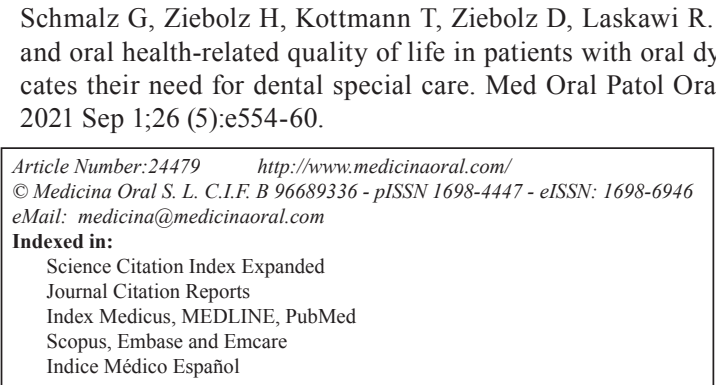

\begin{abstract}
Background: This retrospective study aimed in the evaluation of oral health and oral health-related quality of life (OHRQoL) of patients with oral dystonia (OD).

Material and Methods: Seventeen patients with OD (Meige Syndrome: $n=11$, Oromandibular Dystonia: $n=6$ ) were included, of which seven were examined again at three months after botulinum toxin injection. OHRQoL was assessed by the German short form of oral health impact profile (OHIP G14). Within oral examination, dental parameters, remaining teeth and periodontitis severity were assessed. A matched healthy control (HC) was composed for comparison.

Results: The OD patients had significantly more carious teeth $(0.94 \pm 1.75$ vs. $0 ; p<0.01)$, less remaining teeth $(15.65 \pm 9.89$ vs. $22.22 \pm 5.91 ; p=0.01)$ and higher dental treatment need than the HC ( $42.9 \%$ vs. $0 \% ; p<0.01)$. The OHIP G14 sum score of $9.47 \pm 9.82$ vs. $1.58 \pm 2.79(p<0.01)$ as well its dimensions psychosocial impact $(4.47 \pm$ 6.45 vs. $0.53 \pm 1.16 ; p=0.03)$ and oral function $(4.35 \pm 2.98$ vs. $0.47 \pm 1.34 ; p<0.01)$ were clinically relevant and statistically significant higher in OD compared to HC group. No significant differences could be detected at three months after botulinum toxin injection.

Conclusions: Patients with OD suffer from more dental diseases and have a worse OHRQoL than HC. Dental special care appears recommendable and should be fostered by everyone, who is involved in the treatment of patients with OD.
\end{abstract}

Key words: Oral health-related quality of life, dental health, oromandibular dystonia, meige syndrome. 


\section{Introduction}

Oromandibular Dystonia is characterized as a repetitive and involuntary contraction of the masticatory, facial and/or tongue muscles (1). In case of Meige Syndrom, these symptoms can be complemented by blepharospasm and complex involuntary movement of pharyngeal and cervical muscles (2). The symptoms related to the oral cavity are quite unspecific, reaching from pain, bruxism and tooth fractures to jaw tremor and dislocations/subluxations of the jaw (3). Accordingly, dystonia with oral manifestations are of clinical relevance in dental settings; however, these diseases are rare conditions in dentistry, as a retrospective study over five years showed only 22 patients who entered an orofacial pain clinic to suffer from Oromandibular Dystonia (3). Available examinations in literature have shown a functional impairment and orofacial pain in these patients (3-5). A case series showed beside of problems with mastication, swallowing and hyposalivation an increase in dental problems (4). However, there is up until now no systematic examination of the dental and periodontal health of these patients. Especially data for patients with Meige Syndrome are still completely missing. Under consideration of the functional and psychosocial burden related to these diseases causing oral dystonia (6), a further oral health associated parameter would be of interest for these patients: the oral health-related quality of life (OHRQoL). This parameter describes the perceived impact of oral diseases and conditions on quality of life and can be seen as a sub-aspect of the whole health-related quality of life of a patient (7). Within four domains, the OHRQoL can allow conclusions on functional, psychosocial, pain-related and aesthetic impairments related to the teeth, mouth or dentures (8). Due to its complexity, the OHRQoL of patients suffering from Oromandibu-lar Dystonia or Meige Syndrome would be of relevance.

Up until now, there is no evidence of potential benefit of oral appliances for treatment of oral dystonia (6). How-ever, the injection of botulinum toxin is repeatedly reported to be a promising therapeutic approach (2,5,9-12). Thereby, beside of functional improvements, also an increase in quality of life has been reported (11). In this context, it is unclear whether functional parameters in combination with OHRQoL can be improved by botuli-num toxin, especially over prolonged time period. Furthermore, the oral health situation and a possible necessity of accompanying dental care for these patients is still unclear.

Accordingly, this current study aimed in the evaluation of oral health and OHRQoL of patients with oral dysto-nia (OD) including patients with Oromandibular Dystonia and Meige Syndrome. Furthermore, it should be examined whether a botulinum toxin injection would lead to improved OHRQoL after three months follow- up. For comparison of oral parameters and OHRQoL, a matched healthy control group was included. It was hypoth-esized that patients with OD suffer from worse dental health as well as reduced OHRQoL compared to healthy controls.

\section{Material and Methods}

- Study design

This retrospective study was performed to assess oral health and OHRQoL as well as potential influences of a botulinum toxin injection on OHRQoL in patients with OD, including Oromandibular Dystonia and Meige Syndrome.

- Patients

The records of patients suffering from OD, who attended the Department of Otorhinolaryngology, Head and Neck Surgery, University of Göttingen, Göttingen, Germany between 1st April 2012 and 31st December 2014 were included in the current analysis. All participants were part of a special consultation regarding a therapy with botulinum toxin. The patients provided written informed consent for the analysis of their treatment data at the day of first consultation. At this time point, all participants underwent an oral examination in the Department of Preventive Dentistry, Periodontology and Cariology, University Medical Center Goettingen, Germany by the same experienced dentist (HZ), who was previously trained in performing intra- and extra oral examinations of the patients. If possible, participants were examined again at three months after botulinum toxin injection. To participate in the current study, an age of at least 18 years, presence of an Oromandibular Dystonia or a Meige Syndrome as well as complete oral examination data, including OHRQoL assessment were defined as inclusion criteria. Any exclusion criteria did not exist. For comparison, a healthy control group (HC) consisting of 36 patients with comparable age, gender and smoking habits (matching) was composed from the patients of the Department of Preventive Dentistry, Periodontology and Cariology, University Medical Center Goettingen, Germany. In- and exclusion criteria were equal between groups.

- Oral Health Impact Profile

The German short form of oral health impact profile (OHIP G14) was applied as a validated, questionnairebased tool to assess OHRQoL (13-15). Thereby, 14 questions with regard to functional and psychosocial impacts relat-ed to teeth, mouth or dentures were answered on a five-point scale between $0=$ "never" and $4=$ "always". Accord-ingly, a total score between 0 and 56 points was achievable, with a higher score indicating worse OHRQoL. Beside of statistical significance, clinical relevance was interpreted with regard to the minimal important differ-ence of two points in sum score (16). 
- Oral examination

The oral examination comprised of a dental and periodontal investigation. Thereby, the decayed-, missingand filled teeth index (DMF-T) and the evaluation of periodontal probing depth (PPD) alongside with clinical at-tachment loss (CAL) were elevated. Within DMF-T, the presence of carious teeth with a cavitation of the tooth surface was assigned to the D-T component (17). Moreover, the number of remaining teeth was recorded. Based on PPD and CAL, measured with a millimetre scaled periodontal probe (PCP 15; Hu-Friedy, Chicago, IL, USA) at six measurement points per tooth, the presence of no/mild, moderate or severe periodontitis was evalu-ated (18). If at least one carious lesion, deserving invasive therapy measures was apparent, dental treatment need was rated. In case of a PPD $\geq 3.5 \mathrm{~mm}$ recorded in more than one sextant of the jaw, periodontal treatment need was determined.

Additionally, an examination of the mouth opening (measurement in millimetre) and the evaluation of the axis II Research Diagnostic Criteria for Temporomandibular Disorders (RDC/TMD) (19) was applied at baseline and follow-up for the OD group.

- Statistical analysis

The statistical analysis was performed with SPSS for Windows, version 24.0 (SPSS Inc., US). The metric vari-ables were tested for their normal distribution with Kolmogorov-Smirnov-test. Non-normal distributed samples were analysed by Mann-Whitney-U test. Two dependent variables were analysed by t-test or Wilcoxon test, de-pending on normal distribution, respectively. The comparison between baseline and follow-up data was per-formed with chi-square test, modified after Mc-Nemar. The significance level has been set at $p<0.05$.

\section{Results}

\section{- Patients}

In the OD group, 17 patients were included, of which about two third suffered from Meige Syndrome $(n=11)$ and six patients were diagnosed with Oromandibular Dystonia ( $n=6)$. The HC group consisted of 36 patients with comparable age, gender and smoking habits with the OD group (Table 1). None of the participants had an OD following dental procedures.

- Oral examination

With a value of $22.24 \pm 7.45$, the DMF-T was significantly higher in the OD compared to HC group (17.64 $\pm 5.58, p=0.01)$. Furthermore, the OD patients had significantly more carious teeth $(0.94 \pm 1.75$ vs. $0 ; p<0.01)$ and less remaining teeth $(15.65 \pm 9.89$ vs. $22.22 \pm 5.91$; $p=0.01$ ) than the HC group. Accordingly, the dental treatment need was significantly higher in OD group (42.9\% vs. $0 \% ; p<0.01$, Table 2$)$.

Table 1: Patient characteristics.

\begin{tabular}{|c|c|c|c|c|}
\hline & & OD $(n=17)$ & $\mathrm{HC}(n=36)$ & $p$-value \\
\hline \multicolumn{2}{|c|}{ Gender (male in \% [n]) } & $17.6 \%[3]$ & $16.7 \%[6]$ & 0.99 \\
\hline \multicolumn{2}{|c|}{ Age in years (mv \pm sd [range]) } & $62.47 \pm 14.27[34-78]$ & $62.44 \pm 9.31[44-75]$ & 0.99 \\
\hline \multirow{2}{*}{$\begin{array}{l}\text { Smoking habits } \\
\% \text { [n] }\end{array}$} & smoker & $11.8 \%[2]$ & $13.9 \%[5]$ & \multirow{2}{*}{0.99} \\
\hline & non-smoker & $88.2 \%[15]$ & $86.1 \%[31]$ & \\
\hline \multirow{2}{*}{ Disease \% [n] } & Meige Syndrome & $64.7 \%[11]$ & \multirow[b]{2}{*}{-} & \multirow[b]{2}{*}{-} \\
\hline & Oromandibular distonia & $35.3 \%[6]$ & & \\
\hline
\end{tabular}

OD: oral dystonia, HC: healthy control; significant results $(p<0.05)$ are highlighted in bold

Table 2: Results of the dental findings and OHIP G14 scores between groups.

\begin{tabular}{|c|c|c|c|c|}
\hline & & OD $(n=17)$ & $\mathrm{HC}(\mathrm{n}=36)$ & $p$-value \\
\hline \multicolumn{2}{|l|}{ DMF-T (mv $\pm s d)$} & $22.24 \pm 7.45$ & $17.64 \pm 5.58$ & 0.01 \\
\hline \multicolumn{2}{|l|}{ D-T $(\mathrm{mv} \pm \mathrm{sd})$} & $0.94 \pm 1.75$ & $0 \pm 0$ & $<\mathbf{0 . 0 1}$ \\
\hline \multicolumn{2}{|c|}{ Remaining teeth $(\mathrm{mv} \pm \mathrm{sd})$} & $15.65 \pm 9.89$ & $22.22 \pm 5.91$ & 0.01 \\
\hline \multirow[t]{3}{*}{ Periodontitis (\%[n]) } & No/mild & $35.7 \%[5]$ & $22.2 \%[8]$ & \multirow{3}{*}{0.10} \\
\hline & Moderate & $14.3 \%[2]$ & $47.2 \%[17]$ & \\
\hline & Severe & $50 \%[7]$ & $30.6 \%[11]$ & \\
\hline \multicolumn{2}{|c|}{ Periodontal treatment need $(\%[n])$} & $71.4 \%[10]$ & $77.8 \%[28]$ & 0.72 \\
\hline \multicolumn{2}{|c|}{ Dental treatment need $(\%[n])$} & $42.9 \%[6]$ & 0 & $<0.01$ \\
\hline \multicolumn{2}{|c|}{ OHIP G14 sum $(\mathrm{mv} \pm \mathrm{sd})$} & $9.47 \pm 9.82[4 ; 3.0-16.0]$ & $1.58 \pm 2.79[0 ; 0-2.0]$ & $<0.01$ \\
\hline \multicolumn{2}{|c|}{ Psychosocial impact sum $(\mathrm{mv} \pm \mathrm{sd})$} & $4.47 \pm 6.45[0 ; 0-8.0]$ & $0.53 \pm 1.16[0 ; 0-0.5]$ & $\mathbf{0 . 0 3}$ \\
\hline \multicolumn{2}{|c|}{ Oral function sum $(\mathrm{mv} \pm \mathrm{sd})$} & $4.35 \pm 2.98[3 ; 3.0-6.0]$ & $0.47 \pm 1.34[0 ; 0-0]$ & $<0.01$ \\
\hline
\end{tabular}

OD: oral dystonia, HC: healthy control, mv: mean value, sd: standard deviation, DMF-T: decayed-, missing- and filled-teeth index, OHIP: oral health impact profile, OHIP values are given as mean value \pm standard deviation [median; $25^{\text {th }}-75^{\text {th }}$ percentile]; significant results $(p<0.05)$ are highlighted in bold. 
- Oral health-related quality of life

Within the OHRQoL assessment, OHIP G14 sum score $(9.47 \pm 9.82$ vs. $1.58 \pm 2.79 ; p<0.01)$ as well its di-mensions psychosocial impact $(4.47 \pm 6.45$ vs. $0.53 \pm 1.16$; $p=0.03)$ and oral function $(4.35 \pm 2.98$ vs. $0.47 \pm 1.34$; $p<0.01)$ were clinically relevant and statistically significant higher in OD compared to HC group (Table 2). Furthermore, the majority of questions (9/14) of the OHIP G14 questionnaire were found to be answered significantly worse in OD group ( $p<0.05$, Table 3$)$.
- OHIP G14 and CMD results before and after Botulinum toxin injection

Of the 17 patients with OD, seven patients could be examined again at 3 months after botulinum toxin injec-tion. No significant differences could be detected between baseline and follow-up for OHIP G14 results, mouth opening and Axis II of RDC/TMD ( $p>0.05)$. However, according to minimal important difference, the OHIP G14 sum score was found to be clinically relevant lower at follow-up appointment (Table 4).

Table 3: Results of the OHIP G14 questionnaire.

\begin{tabular}{|c|c|c|c|c|c|c|c|}
\hline \multirow{2}{*}{$\begin{array}{l}\text { Question } \\
\text { [n] }\end{array}$} & \multirow[b]{2}{*}{ Group } & \multicolumn{5}{|c|}{ Point Score } & \multirow[b]{2}{*}{$p$-value } \\
\hline & & $\begin{array}{c}\text { Never } \\
\text { (rating 0) }\end{array}$ & $\begin{array}{c}\text { Rarely } \\
\text { (rating 1) }\end{array}$ & $\begin{array}{c}\text { Sometimes } \\
\text { (rating 2) }\end{array}$ & $\begin{array}{c}\text { Often } \\
\text { (rating 3) }\end{array}$ & $\begin{array}{l}\text { Very often } \\
\text { (rating 4) }\end{array}$ & \\
\hline \multirow{2}{*}{ Trouble pronouncing } & OD & 2 & 12 & 3 & 0 & 0 & \multirow{2}{*}{$<0.01$} \\
\hline & HC & 34 & 2 & 0 & 0 & 0 & \\
\hline \multirow{2}{*}{ Taste worsened } & OD & 4 & 4 & 7 & 2 & 0 & \multirow{2}{*}{$<0.01$} \\
\hline & $\mathrm{HC}$ & 33 & 3 & 0 & 0 & 0 & \\
\hline \multirow{2}{*}{ Life less satisfying } & OD & 13 & 0 & 1 & 3 & 0 & \multirow{2}{*}{0.02} \\
\hline & $\mathrm{HC}$ & 35 & 1 & 0 & 0 & 0 & \\
\hline \multirow{2}{*}{ Difficult to relax } & OD & 16 & 0 & 0 & 1 & 0 & \multirow{2}{*}{0.42} \\
\hline & $\mathrm{HC}$ & 30 & 3 & 2 & 1 & 0 & \\
\hline \multirow{2}{*}{ Feeling of tension } & OD & 10 & 1 & 3 & 3 & 0 & \multirow{2}{*}{$<0.01$} \\
\hline & HC & 34 & 2 & 0 & 0 & 0 & \\
\hline \multirow{2}{*}{ Interrupting meals } & OD & 13 & 0 & 2 & 2 & 0 & \multirow{2}{*}{0.08} \\
\hline & $\mathrm{HC}$ & 34 & 1 & 1 & 0 & 0 & \\
\hline \multirow{2}{*}{ Uncomfortable to eat } & OD & 11 & 2 & 2 & 2 & 0 & \multirow{2}{*}{0.02} \\
\hline & $\mathrm{HC}$ & 34 & 2 & 0 & 0 & 0 & \\
\hline \multirow{2}{*}{ Short tempered } & OD & 13 & 3 & 1 & 0 & 0 & \multirow{2}{*}{0.01} \\
\hline & $\mathrm{HC}$ & 36 & 0 & 0 & 0 & 0 & \\
\hline \multirow{2}{*}{ Difficulty performing jobs } & OD & 12 & 2 & 1 & 2 & 0 & \multirow{2}{*}{0.02} \\
\hline & HC & 35 & 0 & 1 & 0 & 0 & \\
\hline \multirow{2}{*}{ Unable to function } & OD & 13 & 1 & 3 & 0 & 0 & \multirow{2}{*}{0.01} \\
\hline & HC & 36 & 0 & 0 & 0 & 0 & \\
\hline \multirow{2}{*}{ Embarrassed } & OD & 12 & 0 & 4 & 1 & 0 & \multirow{2}{*}{0.01} \\
\hline & $\mathrm{HC}$ & 32 & 4 & 0 & 0 & 0 & \\
\hline \multirow{2}{*}{ Diet unsatisfactory } & OD & 15 & 2 & 0 & 0 & 0 & \multirow{2}{*}{0.74} \\
\hline & $\mathrm{HC}$ & 32 & 3 & 1 & 0 & 0 & \\
\hline \multirow{2}{*}{ Oral pain } & OD & 13 & 0 & 3 & 1 & 0 & \multirow{2}{*}{0.27} \\
\hline & HC & 29 & 3 & 4 & 0 & 0 & \\
\hline Soncef unsortaintu & OD & 16 & 0 & 1 & 0 & 0 & 022 \\
\hline sense or uncertamty & $\mathrm{HC}$ & 29 & 4 & 3 & 0 & 0 & 0.35 \\
\hline
\end{tabular}

OD: oral dystonia, HC: healthy control; significant results $(p<0.05)$ are highlighted in bold.

Table 4: Difference in OHIP G14 and functional findings between baseline and at 3 months after botulinum toxin injection.

\begin{tabular}{|c|c|c|c|c|}
\hline Parameter & Baseline all $(\mathrm{n}=17)$ & Baseline $\mathbf{n}=7$ & Follow-up $(n=7)$ & $p$-value \\
\hline OHIP G14 oral function (mv \pm sd [median]) & $4.35 \pm 2.98[3]$ & $3.57 \pm 1.59[3]$ & $3.43 \pm 0.98[3]$ & 0.99 \\
\hline OHIP G14 psychosocial impact (mv \pm sd [median]) & $4.47 \pm 6.45[0]$ & $4.71 \pm 6.20[1]$ & $2.29 \pm 3.35[0]$ & 0.11 \\
\hline OHIP G14 sum (mv \pm sd [median]) & $9.47 \pm 9.82[4]$ & $9.28 \pm 8.75[4]$ & $6.00 \pm 4.32[4]$ & 0.46 \\
\hline Axis II RDC/TMD sum (mv \pm sd) & $3.06 \pm 2.97$ & $1.86 \pm 2.23$ & $2.43 \pm 3.55$ & 0.34 \\
\hline Painless mouth opening in $\mathrm{mm}(\mathrm{mv} \pm \mathrm{sd})$ & $42.94 \pm 12.0$ & $43.67 \pm 8.03$ & $44.50 \pm 9.12$ & 0.62 \\
\hline Active mouth opening in $\mathrm{mm}(\mathrm{mv} \pm \mathrm{sd})$ & $44.50 \pm 10.44$ & $43.33 \pm 7.43$ & $45.50 \pm 7.79$ & 0.09 \\
\hline Passive mouth opening in $\mathrm{mm}(\mathrm{mv} \pm \mathrm{sd})$ & $47.44 \pm 10.63$ & $47.00 \pm 7.12$ & $47.33 \pm 7.66$ & 0.80 \\
\hline
\end{tabular}

RDC/TMD: research diagnostic criteria for temporomandibular disorders; significant results $(\mathrm{p}<0.05)$ are highlighted in bold. 


\section{Discussion}

- Summary of the main results

Patients with OD in the current study were found to show worse dental health conditions (more caries, less re-maining teeth, higher dental treatment need) than HC. Furthermore, the OHRQoL was clinically relevant and statistically significant worse in OD group.

- Comparison with published data

This is the first clinical study that compared oral health and OHRQoL between a group of OD patients with a HC. While for Meige Syndrom no data are available, yet, some studies have examined patients with Oromandibular Dystonia (3-5). These studies reported on a high prevalence of jaw pain (3) or functional limitations (5). Only one study found a higher number of dental problems in patients with Oromandibular Dystonia (4). Because none of the previous examinations focused on oral health findings, especially caries prevalence, remaining teeth and periodontal conditions, no comparable data for this patient group is available. While the higher caries preva-lence and related dental treatment need as well as less remaining teeth are reported for the first time in the current study, these results might be of clinical relevance. It is known that the number of missing teeth, especially in posterior area, is related to temporomandibular symptoms $(20,21)$. Thereby, an association between missing teeth and clenching as well as chewing difficulties has been reported (20). Therefore, the higher number of miss-ing teeth could be a potential influential factor on functional complaints in OD patients and should be addressed, accordingly. Regarding caries prevalence and dental treatment need, two factors might be related to the findings of the current study. It is known that caries prevalence is related to oral hygiene measures as well as nutritional behaviour $(22,23)$. Caused by psychosocial and functional complaints due to the dystonia of oral muscles, the patients might be less motivated for personal oral hygiene. Furthermore, limited mouth opening or pain might complicate tooth brushing for the patients. On the other hand, due to problems with chewing and swallowing, a balanced nutrition with fruits and vegeTables might be limited, what could increase the risk of caries. Caries can be an important risk predictor for future tooth loss (24), what could lead to more missing teeth and more func-tional complains in the future. Accordingly, patients should receive an intensive dental special care, including individual oral hygiene instructions, information and motivation for personal oral hygiene. Moreover, tooth loss should be prevented and a functional rehabilitation under consideration of respective complaints might be con-sidered.

The OHRQoL of OD group was significantly worse than in HC. With regard to the reference values for healthy German population, i.e. 0-4 points depending on dentition, the OD group can also be seen as worse compared to this reference (25). Thereby, total OHIP G14 sum score and both major dimensions oral function and psychoso-cial impact differed significantly between $\mathrm{OD}$ and $\mathrm{HC}$ groups. All of these differences were also clinically rele-vant, following the principle of minimal important difference (16). The OHRQoL is regularly affected by differ-ent oral conditions; tooth loss or number of remaining teeth, especially remaining functional occlusal pairs are of relevance in this context, respectively $(26,27)$. Therefore, the reduced number of remaining teeth in OD group could affect their OHRQoL. Moreover, temporomandibular disorders can also lead to a remarkable impairment of OHRQoL $(28,29)$. A large German cohort with temporomandibular disorders was found to have an average OHIP G14 of 14.1, what is higher than in the OD patients in the current study (29). Although oral dystonia is different from temporomandibular symptoms in context of craniomandibular dysfunction, a similar effect could be caused by the functional impairment and jaw pain in context of OD. Moreover, psychosocial impacts related to the underlying OD could be a factor that negatively influences patients OHRQoL. Altogether, the reduced OHRQoL appears to be a further argument for dental special care in patients suffering from OD.

A further sub-aspect was the therapy with botulinum toxin injection, which was examined at three months fol-low-up in some of the patients. After three months, seven patients were examined again. Thereby, no significant differences between baseline and follow-up were found. However, the OHIP G14 difference was clinically rele-vant, while missing statistical significance might be caused by the low sample size. No data regarding OHRQoL are available. In general, literature indicates a positive effect of botulinum toxin on complaints of patients with OD $(2,5,9-12)$. In this context, the results of the current study must be seen as preliminary findings, without ability to draw meaningful conclusions on the effect of botulinum toxin injection on OHRQoL of patients with OD.

Altogether, it seems like oral health needs to be supported in patients with OD. It has been stated in literature that dentist needs to be familiar with OD for different reasons; on the one hand, it can develop after dental treat-ment. Furthermore, it is often misdiagnosed as a dental problem and it may cause important functional com-plaints and psychosocial disabilities (30). Based on the current study's findings, dentists' knowledge on OD is also important because dental health and OHRQoL is impaired in these patients. Moreover, otorhinolaryngolo-gists, head and neck surgeons and maxillo-facial surgeons should know about the dental problems of OD pa-tients and the necessity to foster dental care in these individuals. 


\section{- Strengths and limitations}

This current study examined oral health and OHRQoL in a group of patients with OD for the first time. The major limitation is the small sample size. However, the included diseases are rare and therefore a large cohort difficult to recruit. With regard to the small sample size, the findings must be seen as preliminary, especially in the follow-up examination. While the examinations were performed by the same experienced and trained dentist, no blinding procedures for baseline as well as follow-up were performed. A potential selection bias of the in-cluded participants must be discussed. Because all patients were referred for a specific treatment (special consul-tation regarding a therapy with botulinum toxin), the representability of the study cohort remains questionable. The examination did not assess explicitly disease related complaints, which could also impact OHRQoL. OD is heterogeneous in the areas they affect; thereby some of the patients with Meige Syndrome might predominantly suffer from blepharospasm with an unclear impact on oral health and OHRQoL, while these patients could have oromandibular manifestations or not. By assessing this issue more in detail, the potential effect of the different forms of OD on OHRQoL might have been more differentiable. A further methodical limitation is the fact that potential underlying diseases, which might affect oral health (e.g. diabetes, rheumatic diseases) were not recorded and considered within analysis. These co-factors might also influence the results and should be recognized in further studies. Furthermore, the form (jaw-opening vs. jaw-closing) as well as localization/manifestation (e.g. tongue involvement) were not considered and analysed, although these parameters could affect oral complaints in OD patients, what might also be relevant for oral health and OHRQoL of the patients. The assessment of botuli-num toxin at three months after injection might have been suboptimal, as the effect might have been worn of after this period. Thereby it needs to be considered that the applied OHIP G14 retrospectively assessed OHRQoL or related complaints within the previous month, respectively. Additionally, the effect of botulinum toxin on OHRQoL was just a side issue of the current study, while the focus was set at oral health situation of patients with OD to assess their dental needs. Moreover, general healthrelated quality of life issues were not considered in the current study. A multicentric, prospective study which should more comprehensively consider disease related issues would be recommendable to confirm the current study's findings.

\section{Conclusions}

Patients with OD suffer from more dental diseases and show worse OHRQoL than HC. Therefore, dental special care appears recommendable to support patients in their oral hygiene and to prevent caries and tooth loss as good as possible. Everyone, who is involved in the treatment of these patients, including otorhinolaryngologists, head and neck surgeons and maxillo-facial surgeons, should foster their dental care, e.g. by allocation to special dental clinic. The current results and potential benefit of dental special care for patients with OD needs to be validated in prospective studies with a large sample size.

\section{References}

1. Britton D, Alty JE, Mannion CJ. Oromandibular Dystonia: a diagnosis not to miss. Br J Oral Maxillofac Surg. 2020;58:520-4.

2. Pandey S, Sharma S. Meige's syndrome: History, epidemiology, clinical features, pathogenesis and treat-ment. J Neurol Sci. 2017;372:162-70.

3. Sude A, Nixdorf DR. Prevalence and clinical characteristics of patients with Oromandibular Dystonia seen in the orofacial pain clinic: a retrospective study. Oral Surg Oral Med Oral Pathol Oral Radiol. 2020;130:169-74.

4. Bakke M, Larsen BM, Dalager T, Møller E. Oromandibular Dystonia--functional and clinical characteristics: a report on 21 cases. Oral Surg Oral Med Oral Pathol Oral Radiol. 2013;115:e21-6.

5. Bakke M, Baram S, Dalager T, Biernat HB, Møller E. Oromandibular Dystonia, mental distress and oro-facial dysfunction-A followup 8-10 years after start of treatment with botulinum toxin. J Oral Rehabil. 2019;46:441-9.

6. De Meyer M, Vereecke L, Bottenberg P, Jacquet W, Sims AB, Santens P. Oral appliances in the treatment of Oromandibular Dystonia: a systematic review. Acta Neurol Belg. 2020;120:831-6.

7. Reissmann DR, John MT, Schierz O, Kriston L, Hinz A. Association between perceived oral and general health. J Dent. 2013;41:581-9. 8. John MT, Rener-Sitar K, Baba K, Čelebić A, Larsson P, Szabo G, Norton WE, Reissmann DR. Patterns of impaired oral health-related quality of life dimensions. J Oral Rehabil. 2016;43:519-27.

9. Laskawi R, Rohrbach S. Oromandibular dystonia. Clinical forms, diagnosis and examples of therapy with botulinum toxin. Laryngorhinootologie. 2001;80:708-13.

10. Olthoff A, Laskawi R. Zur oromandibulären, pharyngealen und laryngealen Anwendung von Botulinumtox-in [The application of botulinum toxin in oromandibular, pharyngeal and laryngeal dystonia]. HNO. 2012;60:475-8. German.

11. Teemul TA, Patel R, Kanatas A, Carter LM. Management of Oromandibular Dystonia with botulinum A toxin: a series of cases. Br J Oral Maxillofac Surg. 2016;54:1080-4.

12. Pandey S, Sharma S. Botulinum toxin in Meige's syndrome: A video-based case series. Neurol India. 2018;66:71-6.

13. Slade GD, Spencer AJ. Development and evaluation of the oral health impact profile. Community Dent Health. 1994;11:3-11.

14. Slade GD. Derivation and validation of a short-form oral health impact profile. Community Dent Oral Epi-demiol. 1997;25:284-90.

15. John MT, Patrick DL, Slade GD. The German version of the Oral Health Impact Profile--translation and psychometric properties. Eur J Oral Sci. 2002;110:425-33.

16. Reissmann DR. Methodological considerations when measuring oral health-related quality of life. J Oral Rehabil. 2021;48:233-45.

17. Larmas M. Has dental caries prevalence some connection with caries index values in adults? Caries Res. 2010;44:81-4.

18. Eke PI, Page RC, Wei L, Thornton-Evans G, Genco RJ. Update of the case definitions for population-based surveillance of periodontitis. J Periodontol. 2012;83:1449-54

19. Dworkin SF, LeResche L. Research diagnostic criteria for temporomandibular disorders: review, criteria, examinations and specifications, critique. J Craniomandib Disord. 1992;6:301-55.

20. Chatzopoulos GS, Sanchez M, Cisneros A, Wolff LF. Prevalence of temporomandibular symptoms and parafunctional habits in a uni- 
versity dental clinic and association with gender, age, and missing teeth. Cranio. 2019;37:159-67. doi: 10.1080/08869634.2017.1399649.

21. Wang MQ, Xue F, He JJ, Chen JH, Chen CS, Raustia A. Missing posterior teeth and risk of temporoman-dibular disorders. J Dent Res. 2009;88:942-5.

22. Hujoel PP, Lingström P. Nutrition, dental caries and periodontal disease: a narrative review. J Clin Perio-dontol. 2017;44:S79-S84.

23. Andlaw RJ. Oral hygiene and dental caries--a review. Int Dent J. 1978;28:1-6.

24. Haworth S, Shungin D, Kwak SY, Kim HY, West NX, Thomas $\mathrm{SJ}$, et al. Tooth loss is a complex measure of oral disease: Determinants and methodological considerations. Community Dent Oral Epidemiol. 2018;46:555-62.

25. John MT, Micheelis W, Biffar R. Reference values in oral healthrelated quality of life for the abbreviated version of the Oral Health Impact Profile. Swiss Dent J. 2004;114:784-91.

26. Gerritsen AE, Allen PF, Witter DJ, Bronkhorst EM, Creugers NH. Tooth loss and oral health-related quali-ty of life: a systematic review and meta-analysis. Health Qual Life Outcomes. 2010;8:126.

27. Tan H, Peres KG, Peres MA. Retention of Teeth and Oral HealthRelated Quality of Life. J Dent Res. 2016;95:1350-7.

28. John MT, Reissmann DR, Schierz O, Wassell RW. Oral healthrelated quality of life in patients with tem-poromandibular disorders. J Orofac Pain. 2007;21:46-54.

29. Schierz O, John MT, Reissmann DR, Mehrstedt M, Szentpétery A. Comparison of perceived oral health in patients with temporo- mandibular disorders and dental anxiety using oral health-related quality of life profiles. Qual Life Res. 2008;17:857-66.

30. Maestre-Ferrín L, Burguera JA, Peñarrocha-Diago M, Peñarrocha-Diago M. Oromandibular dystonia: a dental approach. Med Oral Patol Oral Cir Bucal. 2010;15:e25-7.

\section{Funding}

The authors declare no grants or financial support.

\section{Conflict of interest}

The authors have stated explicitly that there are no conflicts of interest in connection with this article.

Ethics

The study protocol has been reviewed and approved by the ethics committee of the University Medical Center Goettingen, Germany (No. 14/9/14)

\section{Authors contributions}

Gerhard Schmalz: formal analysis, writing original draft. Holger Ziebolz: data curation, writing review and edit-ing. Tanja Kottmann: formal analysis, wiring review and editing. Dirk Ziebolz and Rainer Laskawi Conceptual-ization, Methodology, Project administration, Writing - review and editing.

Dirk Ziebolz and Rainer Laskawi contributed equally as the senior author. 